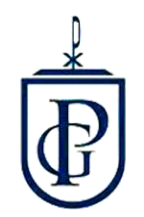

Veritas Et Scientia

Vol. 7, No 1, 781-787

Enero - Junio del 2018.

ISSN $2307-5139$

\title{
EL USO DE LA TECNOLOGÍA Y SU RELACIÓN CON LA CADENA PRODUCTIVA DE LA QUINUA EN LOS AGRICULTORES DE LA LOCALIDAD DE CABANA DE LA REGIÓN DE PUNO, 2016
}

THE USE OF TECHNOLOGY AND ITS RELATION TO THE PRODUCTIVE CHAIN OF THE QUINUA IN THE FARMERS OF THE CABANA LOCALITY OF THE REGION OF PUNO, 2016

\section{Carmen Vicente Avalos Huamani ${ }^{1}$}

\section{RESUMEN}

El objetivo de este trabajo fue conocer la relación existente entre el uso de la tecnología y la cadena productiva de la quinua de los agricultores en la localidad de Cabana en la región de Puno, 2016. La investigación es Básica, con diseño descriptivo. La investigación se desarrolló en el ámbito geográfico de la localidad de Cabana, Provincia de San Román; Departamento de Puno y Región de Puno, en el periodo 2016. La población estuvo compuesta por los pobladores de la zona dedicados a la producción de quinua. La Población analizada está conformada por 2,625 familias-productores de Quinua del Distrito de Cabana/Puno. La muestra es aleatoria y significativa con un tamaño es de 262 familias productoras de Quinua del Distrito de Cabana/Puno. Se trabajó con un cuestionario estructurado. Los resultados de la investigación, demuestran que las familias productoras de quinua, tienen conocimiento adecuado sobre el uso de tecnología, así como de la cadena productiva. Se concluye, que existe relación significativa entre el uso de la tecnología con la cadena productiva de la producción de quinua en los agricultores del Distrito de Cabana, Provincia San Román; Departamento y Región Puno.

Palabras Claves: Quinua, Hábitos agrícolas, ingresos familiares, Tecnología, Cadena productiva, proceso de producción.

(1) Doctor en Administración 


\begin{abstract}
The present investigation has as objective to know the existent relation between the use of the technology and the productive chain of the quinoa of the agriculturists in the locality of Cabana in the region of Puno, 2016. The investigation is Basic, with descriptive design. The research was developed in the geographic area of the town of Cabana, Province of San Román; Department of Puno and Puno region, in the 2016 period. The population was composed by the inhabitants of the area dedicated to the production of quinoa. The analyzed population is made up of 2,625 Quinoa-producing families from the District of Cabana / Puno. The sample is random and significant with a size of 262 families producing Quinoa from the District of Cabana / Puno. We worked with a structured questionnaire. The results of the research show that the quinoa producing families have adequate knowledge about the use of technology, as well as the productive chain. It is concluded that there is a significant relationship between the use of technology with the production chain of quinoa production in the farmers of the Cabana District, San Román Province; Department and Region Puno.
\end{abstract}

Key words: Quinoa, Agricultural habits, family income, Technology, productive chain, production process.

\title{
INTRODUCCIÓN
}

En el Perú las condiciones agroecológicas así como la biodiversidad permiten que se tenga un gran potencial agroalimentario, a causa además del aporte de nuestros ancestros respecto al conocimiento de la producción en flora y fauna; principalmente de aquellos que se producen en la región de la sierra peruana. Es así que debido a la cultura vigente de los pueblos andinos es que la producción de la quinua hoy en día se sigue cultivando. La quinua tiene características únicas: contiene todos los aminoácidos esenciales y es rica en vitaminas, minerales, ácidos grasos y fibra. Con la Resolución № A/RES/66/221, la Asamblea General de las Naciones Unidas, declaró en el 2013, como "Año Internacional de la Quinua", destacándolo como un producto alimenticio de alto valor nutritivo, un cereal que se produce en las tierras de cultivo de la ciudad de Puno en la cuenca del Lago Titicaca, zona originaria de este producto alimenticio. A nivel nacional, el Distrito de Cabana, provincia de San Román, localizado en la región Puno a una altitud media de 3,901 msnm.

Es conocida y reconocida, como la" Cuna y Capital de la quinua en el Perú". El Perú debido a sus microclimas ha sido aventajado en la producción de granos andinos, como la quinua, las condiciones del terreno de cultivo de muy buena calidad hacen posible la calidad del producto. Actualmente en el proceso de producción no se le ha dado la importancia del caso al uso de la tecnología, prima el uso de la mano de obra tradicional, cuestión que en esta zona considerada como la más pobre del Perú no se cuenta, pues todo el proceso de producción se realiza del modo tradicional, que marcaron las condiciones ancestrales, el modo de comercialización del mismo modo, de modo tal que no se ha logrado favorecer la adecuada explotación de este cultivo con fines comerciales y han impedido de esta manera, elevar la calidad de vida de muchas familias peruanas que se han dedicado a la producción de quinua, y que ven en ella un recurso para salir de la extrema pobreza y mejorar su calidad de vida. Una de las necesidades insatisfechas de las microempresas está relacionada a los servicios de financiamiento de mediano y largo plazo en particular para activos productivos como maquinaria y equipos, que son fundamentales para la ampliación de sus negocios, la mejora de su productividad, mayor rentabilidad y el incremento de sus ingresos. La población rural desconoce la importancia del uso de la tecnología por lo que adolece del uso de activos productivos en las fases de producción de la quinua, lo cual limita su productividad, calidad del producto, eficiencia en los costos de producción, esa falta de utilización de tecnología en las actividades agrícolas, hacen del agricultor 
de la cadena productiva de la quinua, vulnerable a permanecer en una economía de subsistencia permanente.

Salcines (Salcines Minaya, 2009) indica la necesidad de incorporar al mercado español a la quinua que se produce en el Perú, apoyándonos en el hecho de que es el producto andino más conocido en este mercado para que comience a ser conocida en el mercado español, hasta llegar a ser consumida. Del Pozo (Arbieto Del Pozo, 2007) afirma que se requiere desarrollar estrategias para la producción comercial de la quinua en el Perú, permitiendo al sector de la quinua ser más competitivo. (Céspedes, 2009), señala que es necesaria conocer las características de la producción, características de la oferta y de la demanda de la misma a fin de darles las características de calidad que requiere el mercado para este cereal. Miranda et al,(Miranda, Carlesso, Huanca, Mamani, \& Borda, 2013) precisan que es necesario investigar las consecuencias que tendría el uso de abono verde en el suelo y evaluar el rendimiento de variedades de quinua, con la finalidad de evaluar la cantidad de biomasa incorporada al suelo. Mamani (Mamani Oroccocllo, 2015), señala que es necesario evaluar la aptitud productiva de doce variedades de quinua (Chenopodium quinoa Willd.), puesto que se requiere conocer el rendimiento de las variedades en agro ecosistema de ladera y planicie y establecer el rendimiento de las variedades en condiciones de cada agro ecosistema.

DE acuerdo a la FAO "La quinua, es el único alimento vegetal que posee todos los aminoácidos esenciales, oligoelementos y vitaminas y no contiene gluten. Los aminoácidos esenciales se encuentran en el núcleo del grano, a diferencia de otros cereales que los tienen en el exosperma o cáscara, como el arroz o trigo". La FAO considera la quinua como uno de los cultivos promisorios de la humanidad, no solo por las propiedades que tiene, si no por los múltiples usos de la planta (Restrepo, Alexandra, Vianchá, \& Peralta Ballesteros, 2005). Los análisis biológicos afirman que la quinua lavada tiene un aporte nutricional de $80.79 \%$ mucho que el maíz, trigo, arroz, centeno, y que la soya.(Mujica et al., 2006). La mayor producción se encuentra precisamente en el distrito de Cabana, en Puno, ciudad sureña del Perú, donde se puede encontrar la mayor cantidad de variedades de la quinua. Es el único distrito donde existe una central de asociaciones de productores, los cuales tienen una planta de procesadora del grano andino pero el índice de expansión de la quinua es muy bajo. Se entiende por cadena productiva de acuerdo a lo que se refiere en el trabajo de Isaza como "un conjunto estructurado de procesos de producción que tiene en común un mismo mercado y en el que las características tecno-productivas de cada eslabón afectan la eficiencia y productividad de la producción en su conjunto" (Isaza-Castro, 2009)(referenciado en Onudi, 2004).

El Proceso de Certificación Orgánica de la quinua, dura aproximadamente tres años, es lo que dificulta a los productores lograr este documento y este proceso se considera como cuello de botella por los productores(«Quinua.pe», 2016). Concepción de la tecnología como un sistema. Como se sabe el factor más versátil de la ciencia y tecnología para la realimentación del conocimiento es la innovación. El punto de convergencia está en el sector industrial, en el que sería imposible para la ciencia, con su propio esfuerzo aislado, crear las bases para obtener nuevo conocimiento sin disponer de la tecnología. Los países que vivimos en el subdesarrollo tenemos que enfrentarnos a una serie de retos para poder competir, lo cual implica realizar una serie de esfuerzos pero en forma mancomunada, para poder lograr fines comunes.

Se requiere de implementar tecnologías que involucren una buena inversión económica, pero que involucre a los productores que como dijimos se encuentran en la región más pobre del Perú y no cuentan con medios económicos para producir a gran escala y para lo cual se requeriría adquirir maquinarias como trilladoras, venteadoras, siendo que la cosecha es una parte del proceso productivo, de ella depende el éxito la obtención de la calidad comercial del grano, esta labor tiene etapas, cuando se efectúa en forma manual o utilizando trilladoras estacionarias: Siega o Corte, Emparvado o formación de arcos, Trilla, Aventado y limpieza del grano y Secado. La educación financiera, más allá de problemas comunes como la informalidad, la marginalidad, los bajos niveles educativos, las barreras lingüísticas, entre otros, estos colectivos enfrentan también otro reto: los asuntos culturales y una cultura financiera inadecuada. 


\section{Objetivos}

a. Conocer el nivel de uso de la tecnología, nivel de conocimiento y dominio de la cadena productiva, en la etapa de la cosecha de la quinua orgánica, en los agricultores de la localidad de Cabana de la región Puno.

b. Identificar el grado de relación existente entre la tecnología y el proceso de la cadena productiva de la producción de la quinua de los agricultores en la localidad de Cabana, en la Región de Puno.

\section{METODOLOGÍA}

Investigación básica, descriptivo correlacional, en el ámbito geográfico de la localidad de Cabana, Provincia de San Román; Departamento de Puno y Región de Puno, en el periodo 2016. Se ha trabajado con los pobladores de la zona dedicados a la producción de quinua. La Población analizada está conformada por 2,785 familias-productores de Quinua del Distrito de Cabana/Puno. La muestra aleatoria tuvo un tamaño de 262 familias productoras. Se trabajó con un cuestionario estructurado, guía Focus group, entrevista a profundidad para productores representativos con seguimiento, control y monitoreo. Para medir y evaluar el grado de confiabilidad de los instrumentos, se considera un Coeficiente de Alfa de Cronbach en una muestra piloto de 20 agricultores de 0.970 .

\section{RESULTADOS}

Respecto al uso de la tecnología en el proceso de la producción de la quinua que en el $69,5 \%$ es adecuado. Respecto al nivel de conocimiento técnico de cómo operar las máquinas para el trillado y el venteo el $54,6 \%$ es muy adecuado. Con un tipo de máquinas para el trillado y venteado de quinua, considerado como adecuado en un $40,1 \%$.

Respecto si utilizaría las maquinarias para el trillado y el venteo de granos de Quinua, en su próxima campaña agrícola, para mejorar su nivel de productividad, el $43,1 \%$ considera esta medida como muy adecuada, pero un $2,7 \%$ como una idea muy inadecuado.

En la cadena productiva de la quinua (tabla 01) se observa que el mayor porcentaje $66,0 \%$ es considerada como adecuada, mientras que el $14,1 \%$ como muy adecuado.

Tabla 01: La cadena productiva

\begin{tabular}{lcc}
\hline Cadena productiva & $\mathrm{n}$ & $\%$ \\
\hline Inadecuada & 52 & 19,8 \\
Adecuada & 173 & 66,0 \\
Muy adecuada & 37 & 14,1 \\
Total & 262 & 100,0 \\
\hline Fuente: Entrevista elaborada & &
\end{tabular}

En referencia a la cantidad de hectáreas de terreno, sólo el 38,9\% considera adecuado esta magnitud. Respecto al costo/kilogramo de quinua (costo de producción) promedio en su campaña, el 50,4\% de los agricultores refieren como aceptable dicho valor, pero sólo el $36,6 \%$ considera como aceptable la rentabilidad por kilogramo que espera obtener en cada cosecha de Quinua.

Respecto a la necesidad de capacitación, el $71,3 \%$ considera como aceptable o muy aceptable y necesario este recurso para todo el proceso de producción. 
Según el uso de tecnología en el proceso de la producción, se observó que el $64 \%$ tiene una adecuada cadena productiva como también el uso de tecnología. Existe relación directa entre la cadena productiva de quinua y el uso de tecnología de los agricultores en la localidad de Cabana en, la Región de Puno. En referencia al tipo de máquinas para el trillado y venteado de quinua, se observó que el $40,1 \%$ considera adecuado este recurso. Existe una relación significativa entre el uso de la tecnología y la cadena productiva (Tabla 02 ).

\begin{tabular}{|c|c|c|c|c|c|}
\hline \multirow{2}{*}{\multicolumn{2}{|c|}{ Cadena productiva }} & \multirow{2}{*}{\multicolumn{3}{|c|}{ Uso de tecnología }} & \multirow[t]{3}{*}{ Total } \\
\hline & & & & & \\
\hline & & \multirow{2}{*}{$\begin{array}{r}\text { Inadecuada } \\
41\end{array}$} & \multirow{2}{*}{$\begin{array}{r}\text { Adecuada } \\
11\end{array}$} & \multirow{2}{*}{$\begin{array}{c}\text { Muy } \\
\text { Adecuada } \\
0\end{array}$} & \\
\hline Inadecuada & $\mathrm{O}_{\mathrm{i}}$ & & & & 52 \\
\hline & $E_{i}$ & 10,3 & 36,1 & 5,6 & 52,0 \\
\hline \multirow[t]{2}{*}{ Adecuada } & $\mathrm{O}_{\mathrm{i}}$ & 3 & 168 & 2 & 173 \\
\hline & $E_{i}$ & 34,3 & 120,2 & 18,5 & 173,0 \\
\hline Muy & $\mathrm{E}_{\mathrm{i}}$ & 8 & 3 & 26 & 37 \\
\hline Adecuada & $\mathrm{O}_{\mathrm{i}}$ & 7,3 & 25,7 & 4,0 & 37,0 \\
\hline \multirow[t]{2}{*}{ Total } & $\mathrm{E}_{\mathrm{i}}$ & 52 & 182 & 28 & 262 \\
\hline & $\mathrm{O}_{\mathrm{i}}$ & 52,0 & 182,0 & 28,0 & 262,0 \\
\hline
\end{tabular}

\section{DISCUSIÓN}

La FAO en la década de los años 70 empleó el concepto de seguridad alimentaria, con el propósito de concientizar a los países que se encuentran en el desarrollo a fin que puedan ayudar con el hambre mundial; definiendo como la responsabilidad de los gobiernos de garantizar el suministro de alimentos en cantidad, calidad y variedad suficientes para proveer de buena salud y nutrición a toda la población. "El centro de origen del cultivo de la quinua es el territorio andino del Perú y Bolivia, desde allí fue llevada por el norte hasta Colombia y por el sur hasta Chile. Su cultivo se realiza, aproximadamente, hace 3,000 a 5,000 años en forma doméstica por los pobladores originarios del altiplano y crece entre $\operatorname{los} 2,500$ y 4,000 m.s.n.m.

Existe problemas en el proceso de la producción de la quinua, desde el modo de cosechar y después de la cosecha de la quinua, los pequeños productores rurales de quinua, trabajan con herramientas artesanales de costumbres ancestrales, haciendo que el trabajo se tedioso para lo que se proyecta hoy en día. De igual manera la forma de secado al sol, y es que en la región no se cuenta con equipos tecnológicos para la trilla ni para la desaponificación, por lo cual estas labores se realizan manualmente, con la participación de varios miembros de la familia.

Por lo general la quinua es comercializada por el agricultor como materia prima pura, sin valores agregados de ninguna clase y por tanto se hace necesario el tener que pasar que pasar por un proceso de beneficiado. En cambio, el agroindustrial comercializa la quinua con valor agregado ofreciendo productos como quinua perlada, hojuelas y otros tanto en el mercado nacional como en el internacional. La presente propuesta permitió comprobar que existe una relación significativa ente el uso de la tecnología y la cadena productiva de quinua, de los agricultores en la localidad del Distrito de Cabana de la Provincia San Román, de la Región de Puno; donde un total de 2,625 microproductores artesanales de la quinua, están interesados, en adquirir las maquinarias para el Trillado y el Venteo; y lograr un cambio tecnológico, basado en el cambio de hábitos agrícolas; es decir, el paso del proceso de la agricultura artesanal-ancestral, a la propuesta innovadora-tecnológica, incorporando 
una tecnología accesible, funcional y vinculante, a la última etapa del proceso productivo de la quinua orgánica; en la etapa de la Cosecha. El resultado de la investigación permite comprobar que la incorporación de la tecnología en la cadena productiva de la quinua, ha logrado que el grano de quinua obtenga una mejor presentación, conllevando a mejores precios por la calidad mejorada del producto, permitiendo mejorar los ingresos y beneficios de los productores, y crear expectativas de proyección a los mercados externos. Pero también existen restricciones críticas identificadas en estudios realizados para el crecimiento y mejora del desempeño de las Pymes rurales, que es el acceso a servicios financieros que se adecuen a sus necesidades permitiéndoles financiar la adquisición de maquinarias para uso en las diversas fases de producción de la quinua. Por lo que es evidente que una de las necesidades insatisfechas de las microempresas está relacionada a los servicios de financiamiento de mediano y largo plazo en particular para activos productivos como maquinaria y equipos, que son fundamentales para la ampliación de sus negocios, la mejora de su productividad, mayor rentabilidad y el incremento de sus ingresos.

En este contexto, acorde a la importancia del uso de la tecnología en la producción de la cadena productiva de la Quinua, se considera que el arrendamiento financiero de pequeña escala o Microleasing para adquirir maquinarias y herramientas, puede ser una alternativa para cubrir las necesidades de financiamiento para inversiones en activos productivos en microempresas, especialmente en áreas rurales, inicialmente como piloto en la provincia de San Román de la región de Puno.

La propuesta de cambio tecnológico, basada en la incorporación de una tecnología accesible y funcional en el proceso productivo de la quinua orgánica, en la etapa de la cosecha, permitió elevar la efectividad de la cadena productiva y la calidad de la producción de la quinua. Mejora de la producción, productividad y la calidad de producto, así como la mejora de los ingresos familiares, reducción de tiempos, y el incremento de los precios de la quinua orgánica, por la calidad mejorada con el proceso tecnológico, así como beneficios de importancia ambiental, cultural, y la tenencia de estos activos productivos, con la posibilidad de alquilarlos, generando ingresos adicionales. El cambio de una agricultura artesanal basada en hábitos agrícolas ancestrales a una agricultura basada en un cambio tecnológico, en el proceso productivo de la quinua orgánica, significa crear nuevas expectativas en la comercialización de la quinua en el mercado interno y externo, así como lograr una transformación en la cadena productiva de la quinua y mejorar sustancialmente los ingresos y beneficios de los productores con bajo nivel de competitividad. Con la incorporación de propuestas de innovación tecnológica en las cadenas productivas de productos agrícolas, en su etapa cosecha, necesariamente va a generar cambios significativos en el proceso de producción y en la calidad del producto final. Es necesario también promover estrategias financieras a través de la Cooperativa de Ahorro y Crédito Cabanillas Mañazo que contribuya con el desarrollo de los procedimientos operacionales y legales que les permitan acceder al producto Micro-leasing a los productores de Quinua y así acceder al uso de tecnología adecuada para lograr una oferta adecuada.

\section{REFERENCIAS BIBLIOGRÁFICAS}

Arbieto Del Pozo. (2007). Quinua Perú. Recuperado 18 de abril de 2018, a partir de https://es.scribd.com/doc/124837768/Arbieto-Del-Pozo-Sheen-Quinua-Peru

Céspedes, R. P. (2009). Digestibilidad in vitro de la proteína y la composición nutricional de tres variedades de quinua (Chenopodium quinoa Willd.) Germinada Y Cocida, 117.

Isaza-Castro, J. (2009). Supply Chains: Approaches and Concepts (Cadenas Productivas: Enfoques Y Precisiones Conceptuales). 
Mamani Oroccocllo. (2015). Efectos de cambio climático en la producción del cultivo de quinua en la zona alta del distrito de llave - el Collao. Recuperado a partir de http://quinua.pe/wp-content/uploads/2016/05/ROGER-MAMANI-OROCCOLLO.pdf

Miranda, R., Carlesso, R., Huanca, M., Mamani, P., \& Borda, A. (2013). Rendimiento y acumulación de nitrógeno en la quinua (Chenopodium quinoa Willd) producida con estiércol y riego suplementario. Venesuelos, 20(1), 21-29. Recuperado a partir de http://saber.ucv.ve/ojs/index.php/rev_venes/article/view/4567

Mujica, A., Ortiz, M. R., Bonifacio, A., Saravia, M. R., Corredor, G., \& Romero, M. A. (2006). Proyecto Quinua: Cultivo Multiproposito para Los Países Andinos, 237.

Quinua.pe, El portal que busca dar a conocer al mundo las maravillosas propiedades de la quinua, producto originario de los Andes. Únete a nuestra comunidad! |. (2016). Recuperado 18 de abril de 2018, a partir de http://quinua.pe/coopain-cabana/

Restrepo, M., Alexandra, L., Vianchá, L. M., \& Peralta Ballesteros, J. (2005). Análisis de variables estratégicas para la conformación de una cadena productiva de quinua en Colombia. Innovar, 15(25), 103-119. Recuperado a partir de http://www.scielo.org.co/scielo.php?script=sci_abstract\&pid=S0121$50512005000100007 \& \operatorname{lng}=e n \& n r m=i s o \& t \operatorname{lng}=e s$

Salcines Minaya, F. (2009). Cadena Agroalimentaria de la Quinua y la Maca Peruana y su Comercialización en el Mercado Español (phd). E.T.S.I. Agrónomos (UPM). Recuperado a partir de http://oa.upm.es/3085/ 